development to evolution is either retrospective or prospective. Failure to keep these two aspects distinct from one another has been the cause of much of the confusion of thought which exists.

The palæontological evidence used in the address is limited to well-authenticated material, made up of numerous specimens carefully collected from known horizons, and is of such a quality that the development of individuals could be worked out with reasonable fullness.

Dealing in the first place with the retrospective aspect of the problem, it is helpful to distinguish between the very early, or embryonic, stages of development and the later, or neanic stages, during which the young gradually assumes the characteristics of the adult. A careful comparison of the development with the successive stages of evolution shows that in some cases there is a remarkably close resemblance between the changes passed through in the neanic period and the changes exhibited by the adults of previous generations. This proves that recapitulation of evolutionary stages as manifested by adults does actually take place. On the other hand, the conditions seen in the embryonic phases exhibit little or no resemblance to any known adult ancestor, but they do resemble very closely the corresponding juvenile stages both of ancestral and of allied forms. This distinction between juvenile and adult recapitulation corresponds with the different bodies of facts observed and emphasized respectively by von Baer and Haeckel, and by their present-day followers.

Though the recapitulation of the complete combination of adult characters seems to be confined to the lower forms of life, the limited recapitulation of individual characters is widely spread even in the highest grades of animals.

Turning to the prospective aspect of the subject, it should be noted that new characters (ccenogenetic) may appear at an early stage of development. Some of these are limited in their occurrence to these stages ; others may extend, in subsequent generations, to later or even adult life. Where this happens, the recapitulatory record may be seriously vitiated. New characters (deuterogenetic) may also appear in the latest stages of development. Such tend, in subsequent generations, to pass back to successively earlier neanic stages, and thus build up a recapitulatory record.

In both these cases the rate at which the new characters attain full expression in evolution varies greatly. In some racial stocks it is attained slowly with the passage of a long period of time. In others the successive phases of expression may come quite rapidly. Occasional instances occur in which the rate of expression appears to be so rapid as to suggest simultaneous mutation. In such cases a false impression of serial evolutionary change is produced by the progressive elimination of individual types in the order of the phases in the series.

In conclusion, it may be suggested that the development of the individual exhibits a body of characters derived from both adult and juvenile stages in the life of its ancestors. This provides a framework into which subsequent evolutionary changes may be fitted. This fitting is carried out by various processes which may proceed separately, simultaneously, or in sequence in the development of different individuals of allied organisms.

\section{Fluctuations in the Abundance of Marine Animals}

IN his presidential address to Section D (Zoology), Dr. Stanley Kemp directs attention to the great fluctuations in the abundance of marine animals and to the need for further study of their causes. Normal annual fluctuations, due for the most part to events which occur in the early stages of the animal's life, may be very great: it is not uncommon to find that one year-class of fish is fifty times as abundant as another. The evidence at present available, drawn from the incidence of good and bad survival years in a few of the more important European fishes, is that these annual fluctuations are strictly localized-a certain species in a particular place will be affected in one way, while another species in the same place, or the same species in another place, will be affected in a different way.

In recent years, it has become increasingly apparent that this is not the only kind of fluctuation to which marine animals are subject. There are others, here called long-period fluctuations, which are superposed upon the annual fluctuations and affect many, or perhaps all, species simultaneously over a wide area.

Two illustrations of such long-period fluctuations are given. In the Channel waters off Plymouth four separate lines of evidence show that since 1931 great changes have taken place. (1) Beginning with 1931, there has been a steady reduction in the numbers of larval fish in the offshore waters : the larvæ of summer-spawning fish have now on the average been reduced to one fifth of their former abundance and those of spring-spawning fish to one third. (2) A marked change has occurred in the constitution of the herring shoals which form the basis of the winter fishery at Plymouth. Since 1931, the proportion of the younger year classes of fish has been reduced from upwards of 66 per cent to less than 20 per cent : in the last 
few years the fishery has suffered greatly and it is now virtually abandoned. (3) In 1930 (and though exact figures are not available it was evidently much the same in former years) the offshore plankton contained Sagitta elegans, which is characteristic of mixed Atlantic water, and S. setosa, which is characteristic of Channel water, in the proportions of $94: 6$; but since 1932, with a single exception in 1936, these proportions have been reversed. (4) The winter maximum of dissolved phosphate has over the same period shown a heavy decrease, amounting on the average to about 35 per cent. Comparable data from other areas are not available, but the failure in recent years of the Donegal herring fishery has been accompanied by a similar change in the constitution of the shoals and may be due to the same causes.

The changes in the Plymouth area are due, it seems, to the lack of influxes of phosphate-rich water from the Atlantic. Salinity and temperature records show, indeed, that incursions of Atlantic water have passed up the Channel ; but the records cannot be correlated with the biological and phosphate data, and the great influx of Atlantic water into the North Sea in 1921 is known to have been detrimental to the herring fisheries. It thus appears that influxes may be of more than one kind, and that only water rich in phosphate, or potentially rich because it carries an abundant plankton, will benefit the Channel fauna.

The second illustration of a long-period fluctuation is from northern waters. Here there have recently been great changes in the fauna, owing to a rise of $1^{\circ}$ or $2^{\circ} \mathrm{C}$. in the temperature of the water, and this is due, presumably, to an increase in the strength of the Atlantic drift. By reason of this change, many animals have been able to extend the limits of their distribution and in almost every section of the fauna significant movements have been noted; profitable cod fisheries have been established at Bear Island and in West Greenland, and an unusual abundance of herring has been found on the Murman coast.

Long-period fluctuations are thus due to a widespread alteration in some hydrographic factor in the environment and though, in the two instances given, this factor is different-at Plymouth phosphate and in northern waters temperature-it is in the ocean to the west that the changes originate and it is here, in the open Atlantic, that the reasons must be sought. At present our knowledge of the circulation in the North Atlantic is very deficient, but with the work now in progress it is to be expected that the main features will shortly be better known. To the biologist, however, it is the irregularities in the system which are of primary importance, and it is only by regular and repeated observations carried out over a wide area that the causes of these long-period fluctuations can be discovered.

In conclusion, attention is directed to the need for greater activity in fishery administration throughout the Empire, for though there are some notable exceptions, it appears that the lessons we have learnt in Great Britain are not generally understood elsewhere. In almost every problem in marine biology it is essential to possess a background of fundamental knowledge, which can only be obtained by long years of patient study. In many parts of the Empire not even a beginning has been made in the acquisition of such knowledge; there are vast areas in which no single species of fish can as yet be recognized in all its stages of development, while little or nothing is known of such matters as rate of growth, spawning periods, food, migrations and fluctuations in abundance. The importance of such information cannot be over-estimated, for the opportunity of dealing effectively with a fishery problem will almost invariably be lost unless knowledge has been obtained in advance and is ready for application.

\section{Correlations and Culture: A Study in Technique}

DROF. GRIFFITH TAYLOR'S presidential address to Section E (Geography) falls into three divisions. In the first the field of cultural geography is considered, in the second a technique which has been found invaluable in that subject is discussed and illustrated ; finally, suggestions are made as to the aspects of culture which should be included in a general education. The relation of geography to history, anthropology, sociology and philology is emphasized. It is shown that there are common fields of research in which the technique of the 'physical' scientist can be used by the cultural geographer to aid in problems in the 'humanistic' disciplines.

The aid geographers can give to historians is illustrated by examples from the Weald and from the blue-grass country of Kentucky. The characteristic cultural development in both is shown to depend on structure primarily, though most historians ignore this important correlation. The use of isopleths (lines of equal abundance) can be used with profit in an interpretation of the essential features of the Renaissance. As an indicator of trends and salient features, they might well be more extensively used by the historian.

The geographer's task should be to teach the student to doubt dogmas involving distribution, and to make his own deductions from fundamental data. In a general education this is a far more valuable function than memorizing economic data, which fills so much of geographic teaching to-day. 